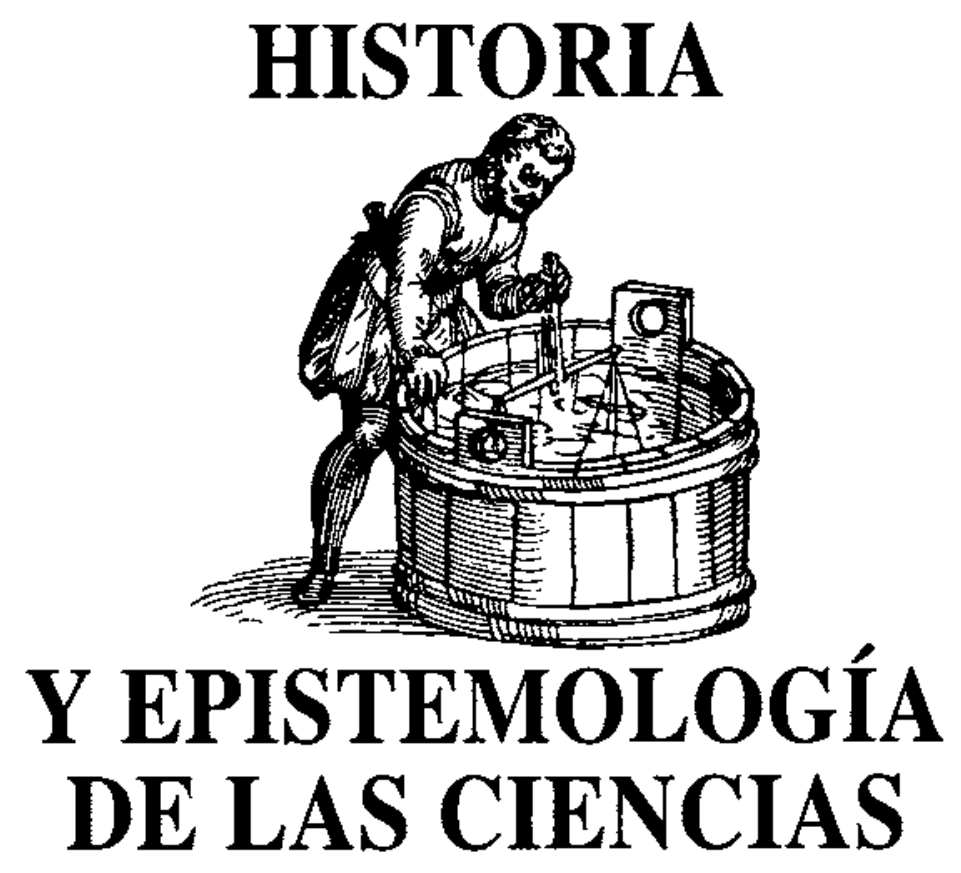

\title{
LA CONSTRUCCIÓN DEL CONCEPTO DE MINERAL: BASES HISTÓRICAS Y UN DISEÑO DE ENSEÑANZA-APRENDIZAJE
}

\section{GALLEGOS, J.A.}

Departamento de Didáctica de Ciencias Experimentales. Facultad de Ciencias de la Educación. Universidad de Granada. 18071 Granada

\section{SUMMARY}

This paper offers a practical model for the teaching-learning of the concept of mineral. It gives information related to the history of the concept. The selection of the suitable material for making this design appropriate to the different levels of the present educational system (or the immediate future one) is left at the choice of each teacher.

\section{PRESUPUESTOS PSICOLÓGICOS Y METO-} \section{DOLÓGICOS}

Si se acepta la base de partida de que los alumnos tienen sus propios esquemas conceptuales y han elaborado sus propias teorías (sus «teorías alternativas») para explicar cómo funciona el mundo (o, al menos, «su mundo»), se 
llega a la conclusión de que el proceso de aprendizaje científico debe consistir en cambiar estas ideas previás por los conceptos que manejan los cientificos (Bruner, Goodnow y Austin, 1978; Vygotsky, 1979).

Por eso, independientemente de que las ideas previas de Ios alumnos tengan las características que les asignaba Driver (1986) o sean más bien apreciaciones menos rigurosas (Pozo, 1993) y hasta distorsiones metodológicas originadas por el propio sistema de exploración (Cubero, 1994), parece imprescindible que el proceso de enseñanza-aprendizaje se base en ellas. La instrucción se deberá diseñar, por tanto, de forma que permita que esas ideas se desarrollen y se cambien por las ideas que son aceptadas por los científicos (Coll et al., 1994). Esto es to que desde el trabajo de Hewson (1981) se ha dado en llamar un cambio conceptual, aspecto que ha dado lugar a numerosos trabajos (Posner et al., 1982; Osborne y Wittrock, 1983). El desarrollo concreto de los postulados propuestos en este sentido (Bachelard, 1938; Driver, Guesne y Tiberghien, 1989; Carrascosa y Gil, 1985; Pozo, 1989; Pozo y otros, 1991) se puede resumir en una serie de «estrategias» concretas como las siguientes:

1) Organizar situaciones en que los alumnos pongan de manifiesto sus ideas.

2) Introducir hechos discrepantes con esas ideas previas.

3) Originar un «diálogo socrático».

4) Estimular la elaboración de esquemas conceptuales correctos.

5) Practicar el empleo de las nuevas ideas en situaciones diversas.

En el caso concreto que nos ocupa, el establecimiento de este concepto debe hacerse, según los currículos actuales, no antes de la ensefianza secundaria obligatoria (ESO), y ampliarlo y precisarlo posteriormente en bachillerato. Por lo tanto, la puesta en práctica del aprendizaje que aquí se sugiere puede resultar más útil si se plantean cuestiones sobre la procedencia mineral de los materiales que nos rodean: desde nuestros propios objetos personales (lápices, estuches metálicos, etc.), los del hogar (vasos de vidrio, ollas y sartenes, cables eléctricos y telefónicos, etc.) y de nuestro entorno social (materiales de construcción, combustibles, cementos, etc.).

Debidamente orientada y coordinada por el profesor, puede resultar una discusión bastante incitadora para despertar el interés por los minerales y las rocas; de ahí se derivará la necesidad de establecer científicamente el concepto mismo de mineral. También se pueden aprovechar, aplicándolas al caso, las sugerencias de Ortega y otros (1993).

De la misma forma, parece extraordinariamente útil echar mano de las enseñanzas de la historia de la ciencia para facilitar la comprensión de los conceptos, como ya múltiples autores han comprobado abundantemente (Saltiel y Viennot, 1985; Gagliardi, 1988; Nielsen y Thomsen, 1990; Hodson, 1994, por ejemplo), e incluso para mejorar la actitud de los alumnos hacia el aprendizaje de los conceptos científicos (Izquierdo, 1994). En este caso concreto, será muy útil y clarificador analizar cómo se ha entendido el concepto en los últimos siglos, las progresivas precisiones y matizaciones, y las actuales diferencias (e incluso discrepancias) entre unos autores y otros, debido a la falta de un consenso total en la valoración de Ios diferentes matices.

\section{SECUENCIA DEENSEÑANZA-APRENDIZAJE}

De acuerdo con todo lo anterior, se propone el esquema ađjunto para orientar la observación, la reflexión y la discusión de los alumnos.

1. Explicitación de ideas previas: Se sugieren, como especialmente indicadas y aprovechables para esta finalidad, reflexiones sobre la utilidad y aplicaciones de los minerales.

2. Seguimiento de la evolución histórica del concepto: Se ofrecen hitos significativos de esa historia.

3. Discusión didáctica de esas concepciones.

4. Elaboración de la definición.

Se pretende con ello, en primer lugar, que se expliciten las ideas previas que los alumnos ya tengan sobre la cuestión; en segundo Iugar, el conocimiento y la reflexión sobre la evolución histórica đel concepto servirá para que comprueben los progresivos esfuerzos realizados por los estudiosos que nos han precedido para ir precisando el concepto de mineral (con los escollos que han tenido que sortear); llegarán a estar así, finalmente, en condiciones de elaborar y proponer una definicion que coincida, esencialmente, con la asumida actualmente en el campo mineralógico.

Desde el punto de vista didáctico, no parece oportuno que la introducción de este concepto se haga antes de los 12-14 años (ciclo $1^{\circ} \mathrm{de}$ ESO) para retomarlo y precisario posteriormente ( ${ }^{\circ}$ ciclo de ESO; bachiller); el nivel de precisión máximo sugerido a continuación debe posponerse hasta después đe los 18 años (Gallegos, 1974, 1977).

Por lo tanto, se deja a juicio del profesor la elección (o selección) de los componentes de este diseño que considere más adecuados, familiares, asequibles, ilustrativos of fácilmente comprensibles para sus alumnos; y esto teniendo en cuenta, tanto su nivel madurativo, como otras características particulares. No obstante, al final, se propone un posible esquema de desarrollo progresivo.

\section{Explicitación de ideas previas}

Me parece especialmente aprovechable comenzar este sondeo y ańlisis de ideas previas en los niveles bajos (ciclo $1^{\circ} \mathrm{de} \mathrm{ESO}$ ), no tanto con preguntas directas ( $i Q u e ́$ piensas que es un mineral?, ¿Qué es un mineral? o similares), como planteando interrogantes sobre el uso, las aplicaciones y el aprovechamiento que han encontra- 
do y siguen encontrando los minerales en las distintas culturas humanas. El rendimiento didáctico que he obtenido desde hace años, parece aconsejar el mantenimiento y acentuación de esta estrategia. Entiendo que, de esta forma, no sólo se consigue el objeto de explicitar contenidos conceptuales, sino que también se aprovechará este aspecto para «llamar la atención» sobre el interés, sobre la importancia, sobre la "necesidad" de ser cons. cientes de nuestras relaciones con el mundo que nos rodea, etc. Es una idea que también parece dar buenos resultados en otros aspectos, corno muestran los trabajos đe Carrillo y Gisbert (1993), Gaopna y Cumbrera (1993), Lillo (1994) y Gil y Valdés (1996), entre otros.

Para ello, como resulta evidente que no se trata de dar una lista exhaustiva de todas las aplicaciones de los minerales, of rezco una serie de sugerencias entre las que me han parecido especialmente ilustrativas y adecuadas. Éstas pueden ser introducidas con preguntas como:

- ¿Comes minerales (alguno por lo menos)?

- ¿Llevas encima algún mineral? ¿Y alguna sustancia de procedencia mineral (obtenida de un mineral)?

- ¿Te pintas con minerales?

$-¿$ Escribes con minerales?

- Etcétera.

\section{- Usos personales y domésticos (adorno, joyería, útiles)}

Haciendo referencia incluso a la prehistoria, se puede comprobar que, desde hace varios milenios, queda constancia del uso de ciertos minerales para estos fines. El sílex (variedad de cuarzo) y la serpentina (junto con rocas como los basaltos y las obsidianas) se utilizaron como raspadores, cuchillos, puntas de flecha y de lanza para la caza (y para la guerra). Por su parte, algunos metales nativos como el cobre, el oro y la plata también llamaron la atención del hombre por su color y su relativa inalterabilidad para obtener objetos de adorno personal y, más tarde, para utensilios caseros. Todavía se sigue utilizando el grafito para fabricar las minas de lápiz (mezclado con más o menos cantidad de arcilla). Igual ocurrió con ciertas «piedras» poco frecuentes, transparentes y de colores llamativos, que acabaron siendo consideradas de especial valor y de especial belleza: a) las dos acepciones de preciosas, como sigue ocurriendo hoy día: diamantes, berilos (especialmente esmeraldas y aguamarinas), corindones («rubíes»); b) semipreciosas: granates, olivinos, turmalinas, zirconitas, topacios, cuarzos, etc.

\section{- Alimentación y medicina (alimentos, conservantes, purgantes, etc.)}

Que la sal común (halita) empezó a apreciarse pronto como necesaria para la alimentación humana y animal, hasta convertirse en objeto de intercambio económico («moneda»), está totalmente comprobado hasta el punto de haber dado lugar a palabras de nuestros idiomas (especialmente «salario»). También debió de ser muy precoz su uso como conservante de pescados y carnes («salazones»). Otros minerales encontraron aplicación en tratamientos de ciertos trastornos humanos, especialmente en forma de revulsivos, eméticos y purgantes. Algunos de ellos han conservado ese uso hasta muy recientemente, en que han sido sustituidos por compuestos muy similares de síntesis artificial. Quizá el ejemplo más llamativo sea el de la sal de La Higuera (pueblo de Madrid), nombre local de un mineral que ha acabado llamándose oficialmente epsomita (sal de Epson). Todavía siguen usándose preparados de yeso, suspensiones de caolín o compuestos de bismuto para el tratamiento de diarreas humanas (mecánicas o infecciosas).

\section{- Decoración (pinturas, incrustaciones, mosaicos)}

Algunos minerales coloreados, que presentan facilidad para pulverizarse y originar suspensiones acuosas (como hematites, pirolusita, antimonita, etc.), ya fueron utilizados hace más de diez mil años como pigmentos para pintar (pinturas rupestres), o para pintarse (cosmética humana). Más tardíamente se amplió esta gama de colo. res con azuritas y malaquitas, cinabrio, azufre y otros. Igualmente, los trocitos de tamaños milimétricos o centimétricos también encontraron aplicaciones como piezas de mosaico junto a otros trozos de rocas o en incrus* taciones sobre madera, hueso o metales; entre ellos se encuentran muchos de los anteriores y algunos más como la lazurita, el ópalo, distintas variedades de calcedonia, etc.

\section{- Agricultura (modificación de suelos, abonos, pla* guicidas)}

En la misma línea anterior, pero aplicada en sentido contrario, como venenos para ciertas especies molestas o dañinas para el hombre, se encuentran minerales con arsénico (rejalgar, oropimente, sulfoantimoniuros, etc.) o con cobre (sulfato de cobre) especialmente; encuentran uso como insecticidas y fungicidas. Empíricamente se descubrió, ya en el neolítico tardío, una vez bien asentada la agricultura, al menos en el dominio mediterráneo, la necesidad de «corregir» la composición del suelo, añadiendo calizas a los muy arcillosos, arcillas a los muy calizos, i otros. Muy posteriormente, consolidado durante siglos el éxito (igualmente empírico) de los abonos orgánicos («estiércol»), se inició el abonado con sustancias naturales pero minerales, de las que quizá el primer ejemplo sea el nitrato de Chile; ello está relacionado directamente ya con el estudio y descubrimiento del papel de los abonos artificiales (fosfatos, sulfatos, nitratos, etc.) y de los oligoelementos.

\section{- Metalurgia (cobre, estaño, oro, plata, hierro, alu- minio, plomo, etc.)}

También es muy antiguo el descubrimiento de la extracción de ciertos metales; sobre todo aquéllos que forman 
parte de minerales de los que no resulta especialmente dificil separarlos, básicamente, por su bajo punto de fusión, como son el cobre y el estaño. Cuando se extendieron estos procesos, conocidos como metalurgia, se considera comenzada, en la prehistoria, la edad de los metales: la edad del cobre, la edad del bronce (aleación de cobre y estaño), la edad del hierro. Justamente el hierro (el más difícil de extraer de los citados porque requiere temperaturas de fusión más altas a la vez que un ambiente reductor adecuado) sigue siendo un metal imprescindible en la actualidad (seguimos en la edad del hierro), aunque sea más o menos modificado en forma de aceros (hierro con una pequeña cantidad de carbono disperso $\mathrm{y}$, últimamente, con otros elementos también -cromo, vanadio, wolframio, etc.-, que constituyen los aceros especiales). No obstante, desde mediados del siglo XX se ha ido incrementando el uso del aluminio, en forma también de «aluminios especiales» (en aleaciones similares a las de los aceros especiales citados), que está sustituyendo con ventaja al hierro y al acero, sobre todo, por su menor densidad, aunque también por su mayor conductividad eléctrica (redes de alta tensión).

\section{Breve resumen sobre la evolución histórica del concepto}

Para proporcionar la base histórica en la que fundamentar una discusión del concepto de mineral que hoy día se maneja, y dar a los alumnos un bosquejo histórico de su evolución, parece conveniente limitarse a la edad moderna, puesto que es desde entonces cuando se encuentra en un campo suficientemente preciso y delimitado (separado ya de los fósiles, a los que se ha encontrado unido hasta entonces como materiales pétreos naturales). Así pues, se pueden suministrar a los alumnos reseñas que contengan la información (mandarlos a buscarla, aunque sea orientándolos, puede ser excesivo para la mayoría de los alumnos y de las bibliotecas de los centros). Estas reseñas tendrán que ser muy seleccionadas (aun cuando se deje la discusión de este aspecto histórico para el nivel universitario), espigando los hitos más sobresalientes, aun a riesgo de que resulte un conjunto pobre, parcial o subjetivo; parece obligado, por un lado, para no perderse en un bosque excesivamente denso de información; por otro, para que no requiera un tiempo de desarrollo claramente desproporcionado en el disponible para los programas correspondientes (también se deja a juicio del profesor, en consecuencia, escoger sólo algunos de los hitos que aquí se ofrecen).

En primer lugar hay que citar a Agrícola (1546), llamado realmente Georg Bauer. En su primera obra, De natura fossilium, queda patente que bajo ese nombre se englo. ban todos los materiales que se extraen del subsuelo, independientemente de su origen o de su composición; de hecho, él prácticamente sólo se ocupa de los minerales de interés económico. Igual ocurrirá con su obra magna (publicada póstumamente) y mucho más erudita, De re metallica (1556). Se nota todavía la influencia de Plinio (c 75), que es el primero en utilizar la palabra fossilis (derivándola del verbo fodio-fodere-fossum = cavar) para designar «lo que se saca cavando de la tierra» (piedras, tierras, minerales, etc.).

Palissy (1580) aporta ya precisiones interesantes sobre la idea que más tarde acabará consolidándose conceptualmente bajo el término de mineral, separándolo claramente de los materiales se procedencia orgánica, aunque estén mineralizados (que corresponderían ya al concepto moderno de fósil). Pero resulta claro que hay que esperar a Stenon ( 1669) para encontrarse con una aproximación suficientemente elaborada del concepto que pueda ser considerada como punto de partida del concepto actual. Efectivamente, es entonces cuando se propone una explicación justificada de la formación de minerales en el seno de disoluciones, como consecuencia de lo que hoy día entendemos como «precipitación» de una disolución.

En una línea similar se coloca Werner (1791), en lo que respecta al concepto, si bien su apriorismo de la formación de los minerales «por descenso» de soluciones de sustancias disueltas en agua u otros compuestos (ácido sulfúrico concretamente para la formación de los carbones) condiciona el resto de sus interpretaciones sobre los filones y la génesis mineral en general.

El uso del término con una limitación similar a la que presenta actualmente (separado ya de los fósiles y de las rocas) parece que sólo se alcanza más tarde, aunque resulte difícil precisarlo totalmente. En cualquier caso, es en la obra de Monnet (1779) donde sí se encuentra el término mineralogía (la ciencia que estudia los minerales) como un concepto totalmente consagrado $y$ extendido.

Resulta curioso, sin embargo, que a finales de siglo XVIII, un autor español (Del Río, 1795), introductor en España e Hispanoamérica de las ideas de Werner, todavía utiliza el término fósiles para designar tanto a los minerales como a los fósiles en sentido actual; justamente llama a estos segundos figuras extrañas, porque considera como «figuras propias» a los auténticos minerales.

A partir de ese momento, independientemente de que sigue prestándosele abundante atención a las menas metálicas (por su evidente interés aplicado), el análisis teórico parece centrarse más bien en la explicación de la génesis de los cristales y de las razones por las que la materia se organiza de esa forma poliédrica tan liamativa. Hitos importantes en esa línea son el mismo Stenon, Hauy y Bravais, antes de llegar al siglo XX, que será cuando se conseguirán las precisiones definitivas para la elaboración actual del concepto.

Desde un punto de vista histórico sería interesante analizar si, precisamente, la necesidad de establecer la estructura interna de la materia mineral (en la que se concentra la atención durante todo el siglo XIX) es la razón de que se estanque la maduración del concepto. Desde un punto de vista epistemológico-didáctico, sería igualmente ilustrativo comprobar si es la necesidad de comprender la discontinuidad de la materia y su organi- 
zación espacial la que origina dificultades de comprensión de los conceptos que presenten ese tipo de implicaciones en nuestros alumnos (como el autor, personalmente, estima, con los análisis que ya realizó sobre este aspecto (Gallegos, 1974).

En el siglo XX son ya abundantísimos los autores especializados en mineralogía que incluyen una definición del concepto de mineral e, incluso, una discusión de su alcance, sus limitaciones o sus implicaciones. Por lo tanto, se citan sólo unos pocos, muy seleccionados, que se consideran suficientemente representativos. Esta selección la he realizado de forma que además cubra también diferentes campos de especialización; queda así ocasión para discutir si las diferencias que se aprecian pueden ser achacadas al enfoque de la obra en función de los previsibles lectores.

En primer lugar cabe citar el Diccionario Enciclopédico Espasa (1930), que puede ser considerado claramente representativo de las ideas reinantes en el primer cuarto de nuestro siglo. Dice (t. 35, p. 524):

«Los materiales constitutivos de la corteza terrestre pueden agruparse cientificamente en tres secciones, minerales, rocas y fósiles, que son estudiadas separadamente en tres de las ciencias derivadas de la Geología: Ia Mineralogía, la Petrografía y la Paleontología. Se llama minerales a aquéllos de dichos materiales que tienen composición química definida o forma geométrica determinada.»

Sin embargo, unas cuantas páginas más adelante, cuando se trata de definir mineralogía (t. 35, p. 563), se presenta una idea no totalmente coherente con lo anterior, pues dice:

«[...] que se ocupa de los minerales, esto es, los cuerpos no orgánicos y los orgánicos no organizados que entran a formar parte de la corteza terrestre».

Más recientemente, Klockmann (1961, p. 1), en un tratado clásico de mineralogía y de extraordinario prestigio durante varias décadas, ofrece la siguiente definición, que puede considerarse la asumida en su campo específico hasta ese momento:

«Bajo la denominación de minerales comprendemos los materiales elementales, de origen natural, que constituyen la corteza sólida de la Tierra».

En la Mineralogía de Kostov (1968), aparece la siguiente definición:

«Un constituyente natural y homogéneo de la corteza terrestre que posee un composición química definidida y una estuctura cristalina estable dentro de ciertos Í́mites físico-químicos.»

La traducción española del Manual de Mineralogía de Dana, actualizada por Hurlbut, también parece muy usada por los alumnos que tienen que ocuparse de estos temas a nivel universitario. En la edictón de 1978, en las páginas introductorias, después de justificar la necesidad de restringir el estudio a las sutancias naturales y de recordar que los minerales pueden ser sustancias simples o compuestas, of rece la siguiente definición (p. 2):

«Elemento o combinación química formada mediante un proceso inorgánico natural.»

En la edición de 1993 (21a.) aparecen variantes significativas respecto de la anterior:

«Un sólido natural homogéneo con composición química definida (pero generalmente no fija) y una ordenación atómica muy alta, que se forma normalmente por procesos inorgánicos.»

Finalmente, he recogido las definiciones propuestas en los dos diccionarios de geología más completos publicados en español; están trađucidos, uno del inglés y otro del francés, y parecen haber encontrado una difusion importante (como yo mismo he tenido ocasión de comprobar).

Whitten y Brooks (1980, p. 180) dice:

«Sólido estructuralmente homogéneo de composición química definida formado por los procesos inorgánicos de la naturaleza.»

Y Foucault y Raoult (1985, p. 197):

«Especie química natural que se presenta la mayoría de las veces en forma de sólido cristalino.»

\section{Discusión didáctica}

Desde un punto de vista didáctico se propone seguir dos caminos, en función del nivel de los alumnos. Para los alumnos de ESO, se partirá de sus propias definiciones, para hacerles tomar conciencia de las vaguedades $e$ imprecisiones que presentan, solicitándoles que afinen la expresión del concepto, pero dejándolo abierto a posteriores revisiones (relacionadas con la progresión de sus conocimientos). Para los de bachiller, parece adecuado partir, no sólo de las revisiones anteriores, sino también de la comparación con las definiciones que se encuentran en los libros recientes, haciéndoles tomar conciencia de las importantes consecuencias que la definición que adoptemos implica en la elaboración de conceptos muy significativos en mineralogía, como diferencias entre mineral y roca (sobre todo si se trata de rocas monomineralicas), variedades minerales $\mathrm{y}$, especialmente, polimorfismo e isomorfismo (y series isomorfas).

Será, pues, en bachiller (preferiblemente en $2^{\circ}$ curso) cuando puede resultar útil plantear a los alumnos preguntas como:

$-¿$ Qué parecidos hay entre la definición propuesta por el grupo y la que ofrece Klockmann?

$-¿$ Qué diferencias? 
$-¿$ Qué materiales naturales quedan incluidos en una y en otra?

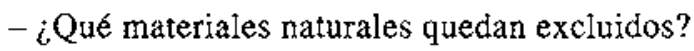

- Entre la definición de Klockmann y la de Kostov, ¿qué parecidos y qué diferencias hay?

- Compara esas definiciones con la de la Enciclopedia Espasa: ¿Qué diferencias aprecias? ¿Qué importancia te parece que tienen esas diferencias?

$-{ }^{-}$Qué matiz nuevo introduce la definición de Whitten respecto a las anteriores?

\section{- Etcétera.}

El objeto es que reflexionen sobre detalles o matices similares a los que recojo a continuación, que deben permitirles ir clarificando y precisando sus ideas.

Por ejemplo, en la definición de la Enciclopedia Espasa, en el primer párrafo llama la atención que se establezca la disyuntiva entre una composición «o» una forma geométrica determinada; otros autores, ya en esa época, exigían las dos condiciones juntas. En la misma definición, a continuación, parece sugerirse que la negación de organización para los cuerpos orgánicos se refiere a una organización biológica, es decir, una organización en tanto en cuanto organismos vivos o restos muertos de organismos vivos; pero se presta a confusión con la exigencia anterior de que los minerales deban tener una forma geométrica definida. Además, en esta ségunda formulación no se exige una composición química, ni constante, ni determinada, como había ocurrido en la primera; en ésta se había comentado y matizado este aspecto, justificando la expresión composición química definida como distinta y menos exigente que la de composición química constante, para dejar lugar a la existencia de impurezas y a la aparición de variedades de tipo químico, dentro de la misma especie mineral.

En el caso de Klockmann, es verdad que, comentando la definición, hace precisiones sobre la composición quf́mica y la estructura, pero resulta llamativo que no las incluya en la definición misma, lo cual puede ser considerado un defecto de forma. Por otra parte, el referirse a los minerales como «materiales elementales» introduce una grave colisión con los conceptos químicos; en es:a rama de la ciencia, el concepto de elemento tiene una significacion totalmente precisa (sustancias formadas por un solo tipo de átomos), que no encaja bien con un uso vulgar del término en una definición científica. Finalmente, habría que quitar la limitación referida a la corteza de la Tierra, porque también pueden existir o provenir de capas más internas del planeta (o incluso de otros astros).

La definición de Kostov parece muy asumible, salvo por Io que respecta a la restricción sobre la corteza terrestre, que ya se queda claramente estrecha. Así, por ejemplo, al decir solamente natural, no impide que pueda ser de origen orgánico. Al dẹcir homogénen implica que no presente cambios de pureza, sobre todo, tan próximos o acusados que pueda confundirse con una roca monominerálica. Al añadir «estructura cristalina estable dentro de ciertos límites físico-químicos» sale al paso de los posibles problemas relacionados con transiciones polimorfas.

La definición de Dana-Hurlbut (1978) recoge la exigencia de "natural» y la de proceso genético inorgánico, pero no incluye, expresamente, la exigencia de cristalinidad (ordenación espacial de las partículas materiales); ésta la comenta dentro del significado que le da a la exigencia de que sean elementos o compuestos químicos, lo cual me parece menos adecuado que su alternativa (la inclusión expresa de esta condición); por su parte, la constancia química parece quedar expuesta de forma menos expresiva de la que parecería conveniente. Algunas de estas observaciones (que parece haberlas hecho también el autor, por su parte) pierden su valor al aplicarlas a la definición de 1993; ésta ya sí incluye la ordenación atómica (aunque aparece otro escollo al exigir que ésta sea muy alta).

En la definición de Whiten y Brooks, se ven claramente las condiciones impuestas:

a) que sea estructuralmente homogéneo;

b) que sea de composición química definida (término que justifica el propio autor a continuación para subrayar que no se trata de sustancias puras, sino que se acepta un cierto margen de impurezas);

c) que sea formado por procesos inorgánicos;

d) que sea natural (de la naturaleza).

Sin embargo, es necesario llamar la atención sobre la imprecisión de la característica cestructuralmente homogéneo» porque, en principio, una materia amorfa, es decir, con las partículas distribuidas aleatoriamente, también es estructuralmente homogénea y no queda excluida, en este caso, porque no aparece ninguna restricción referida a la cristalinidad.

En la definición ofrecida por Foucault y Raoult, finalmente; son diferentes los requisitos:

a) que sea una especie química;

b) que se presente en forma de sólido cristalino;

c) que puede no presentarse en forma de sólido cristalino.

En este caso, el requisito a no está bien expresado, puesto que no puede entenderse en sentido restringido (= sustancia pura); es verdad que algunos minerales son sorprendentemente puros (sobre todo los que se presentan como elementos nativos), pero es igualmente cierto que los compuestos, prácticamente siempre, contienen una cantidad de impurezas que impiden considerarlos como sustancias químicamente puras. Por su parte, el requisito $b$ es algo confuso porque queda la duda de si se 
está refiriendo a una forma poliédrica (cristal), tal como sugiere la expresion la mayoria de las veces; en $c$ las veces en que no es sólido cristalino, no queda claro si debe seguir pensándose que sea materia cristalina (partículas ordenadas en el espacio), aun cuando la ordenación interna no se manifieste al exterior en forma poliédrica.

\section{CONCLUSIÓN}

Como consecuencia de todo lo expuesto, debe surgir la necesidad de elaborar una defínición que:

a) trate de englobar y aglutinar todos los aspectos y matices parcialmente considerados en las definiciones anteriores;

b) soslaye Ios inconvenientes detectados anteriormente;

c) haga justicia a los avances conseguidos en los estudios estructurales realizados en el análisis de los minerales desde los descubrimientos de Laue (que se consideran esenciales desde hace décadas).

De esta forma, los alumnos de los últimos cursos de ESO o del nuevo bachiller pueden entender dos aspectos fundamentales para la comprensión de los conceptos científicos y de la ciencia misma:

a) que las definiciones científicas son provisionales, por cuanto representan el estado del conocimiento en el momento en que son formuladas;

b) que en esas formulaciones intervienen siempre, aunque en diversa medida, las distintas valoraciones que cada autor hace de los diferentes matices implicados.

No obstante, podrán llegar a proponer, defendiéndola y justificándola, aun con clara conciencia de su provisionalidad, una definición de mineral más o menos similar a la siguiente:

«Sustancia natural homogénea originada por un proceso geologico, de composición química definida dentro de uri estrecho margen y cristalización fija.»

Para conseguir este desarrollo de profundización y matización progresiva parece necesario partir de aspectos muy concretos y avanzar paulatinamente hacia niveles más complejos y abstractos. Se propone, pues, Ia siguiente secuencia, tomando como referencia la edad cronológica de los alumnos, siguiendo las conclusiones claramente establecidas por Piaget (1970), con independencia de que haya desfases verticales y horizontales, como muchos autores han puesto de manifesto (Flavell, $1977 \mathrm{y}$, especialmente en el campo concreto de las ciencias (Shayer y Adey, 1986; Hierrezuelo y Montero, 1991); pero, de todas, formas, resulta una referencia necesaria y es más adecuada que sus alternativas (EGB, primaria, ESO, etc.) por cuanto que está centrada en el alumno y no en diseños curriculares que responden más a criterios socioculturales o de estrategia política.

\section{2-14 años}

Explicitación de ideas previas: A través de preguntas motivadoras como las sugeridas anteriormente, se puede terminar concretando qué consideran los alumnos como mineral.

Discusión didáctica: Conseguir una detección y toma de conciencia de las incoherencias e inconsistencias entre el concepto expresado de mineral y otros conceptos relacionados con él (por ejemplo: roca, elemento químico, mena, etc.).

Elaboración de una definición más aproximada de mineral.

\section{4-16 años}

Explicitación de ideas previas: Proceso similar al anterior.

Discusión didáctica: Proceso similar al anterior.

Definiciones científicas: Introducir alguna definición bien escogida de las propuestas en los textos y repetir la discusión didáctica anterior cn estos nuevos elementos de juicio.

Elaboración de una definición más aproximada de mineral.

\section{6-18 años}

Explicitación de ideas previas: Proceso similar al anterior.

Discusión didáctica: Análisis comparativo de «nuestra» definición y las existentes en la bibliografía científica propuesta. Crítica de una y otras.

Breve revisión histórica: Introducir algunos hitos señeros de la evolución histórica, reflexionando y discutiendo las modificaciones y avances implicados.

Asunción justificada de una definición científica de mineral.

\section{Universidad (ciclo $1^{\circ}$ )}

Explicitación de ideas previas: Proceso similar al anterior.

Discusión didáctica: Análisis de las progresivas modificaciones históricas del concepto. Justificación de los sucesivos cambios e introducciones motivados por los nuevos descubrimientos.

Comprensión y justificación crítica de la definición cientifica actual.

A modo de resumen, se representa esquemáticamente el diseño anterior en el siguiente cuadro: 
Tabla I

Esquema de secuencia progresiva para el aprendizaje del concepto mineral.

\begin{tabular}{|c|c|c|c|c|c|}
\hline Años & Ideas previas & Revisión histórica & Definición científica & Discusión didáctica & Conclusión \\
\hline $12-14$ & Deteccion & & & $\begin{array}{l}\text { Reflexión crítica } \\
\text { y corrección de } \\
\text { incoherencias }\end{array}$ & $\begin{array}{l}\text { Definicion } \\
\text { revisada }\end{array}$ \\
\hline 14-16 & Detección & & & $\begin{array}{l}\text { Reflexión crítica } \\
\text { y corrección de } \\
\text { incoherencias }\end{array}$ & $\begin{array}{l}\text { Definición } \\
\text { refinada }\end{array}$ \\
\hline $16-18$ & Deteccion & & $\begin{array}{l}\text { Algunas definiciones } \\
\text { cientifitcas }\end{array}$ & $\begin{array}{l}\text { Reflexión crítica y } \\
\text { corrección de } \\
\text { incoherencias. } \\
\text { Discusión razonada } \\
\text { de una definición } \\
\text { científica }\end{array}$ & $\begin{array}{l}\text { Definición } \\
\text { actual }\end{array}$ \\
\hline$>18$ & Deteccion & $\begin{array}{l}\text { Definición de cada } \\
\text { spoca en relación } \\
\text { con el estado } \\
\text { del conocimiento }\end{array}$ & $\begin{array}{l}\text { Justificación de los } \\
\text { cambios y progresivas } \\
\text { matizaciones en las } \\
\text { definiciones científicas }\end{array}$ & $\begin{array}{l}\text { Reflexión crítica } \\
\text { y refinamiento } \\
\text { de la definición }\end{array}$ & $\begin{array}{l}\text { Definición } \\
\text { actual }\end{array}$ \\
\hline
\end{tabular}

\section{REFERENCIAS BIBLIOGRÁFICAS}

ADAMS,F.D. (1954).The birthand development of the geological Science. Nueva York: Dover Publ.

AGRICOLA (BAUER, G. (1546). De natura fossilium. (Resumido en Adams, 1954, pp. 191-195).

AGRICOLA (BAUER, G. (1556). De re metallica. Halleux, R. y Yans, A. (eds.). 1990. Les Belles Lettres.

BACHELARD, G. (1938). La formation de l'esprit scientifique. París: Vrin.

BRUNER, J.S., GOODNOW, I. y AUSTIN, G.A. (1978). El proceso mental en el aprendizaje. Madrid: Narcea.

CARRASCOSA, J. y GIL, D. (1985). La metodología de la superficialidad y el aprendizaje de las ciencias. Eneñanza de las Ciencias, 3(2), pp. 113-120.

CARRILLO, L. y GISBERT, J. (1993). Pero... ¿ hay rocas en la calle? Servicio de Medio Ambiente del Ayuntamiento de Zaragoza.

COLL, C. et al. (1994). El constructivismo en elaula. Barcelona: Graó.

CUBERO, R. (1994). Concepciones alternativas, preconceptos, errores conceptuales... ¿distinta terminología y un mismo significado? Investigación en la Escuela, 23, pp. 33-42.
DANA, A. y HURLBUT, C.S. (1978). Manual de mineralogía. Madrid: Reverté.

DANA, A. y HURLBUT, C.S. (1993). Manual de mineralogia. Madrid: Reverté.

DEL RIO, A.M. (1795). Elementos de orictognosia o del conocimiento de los fósiles... Zúñiga y Ontiveros. Méjico. (1985, ed. facsímit. Univ. Compl. Madrid).

DRIVER, R. (1986). Psicología cognoscitiva y esquemas concetuales de los alumnos. Enseñanza de las Ciencias, 4(1), pp. 3-15.

DRIVER, R., GUESNE, E. y TLBERGHIEN, A. (1989). Ideas cientificas en la infancia y la adolescencia. Madrid: MorataMEC.

DICCIONARIO...(1930). Diccionario Enciclopédico. Madrid: Espasa-Calpe.

FLAVELL, J.H. (1977). Cognitive depelopment. Nueva Jersey: Prentice Hall.

FOUCAULT, A. y RAOULT, J.F. (1984). Diccionaire de Géologie. Paris: Masson.

FOUCAULT, A. y RAOULT, J.F. (1985). Diccionario de Geología. Barcelona: Masson. 
GAGLIARDI, R. (1988). Cómo utilizar la historia de las ciencias en la enserianza de las ciencias. Enseñanza de las Ciencias, 12(2), pp. 188-199.

GALLEGOS, J.A. (1974). La cristalografía en el bachillerato. La Educación Hoy, 2(1), pp. 27-30.

GALLEGOS, J.A. (1977). Claves mineralógicas. Granada: Tall. Arte.

GAONA, A. y CUMBRERA, F. (1993). Las rocas omamentales. Sus aplicaciones didácticas. Enseñanza Ciencias Tierra, 1(1), pp. 19-25.

GIL, D. y VALDEZ, P. (1996). La orientación de las prácticas de laboratorio como investigación: un ejemplo ilustrativo. Enseñanza de las Ciencias, 14(2), pp. 155-163.

HEWSON, P. (1981). A conceptual change approach to learning Science methodological change». Eur. J. Sc. Education, pp. $12,25-57$.

HIERREZUELO, J. y MONTERO, A. (1991). La ciencia de los alumnos. Vélez Málaga: Elzevir.

HODSON, D. (1994). Hacia un enfoque más crítico del trabajo de laboratorio. Enseñanza de las Ciencias, 12(3), pp. 299 313.

IZQUIERDO, M. (1994). ¿Cómo contribuye la historia de las ciencias en las actitudes del alumnado hacia el aprendizaje de las ciencias. Aula Inv. Educativa, 27, pp. 37-40.

KLOCKMANN, F. y RAMDORHR, P. (1661). Tratado de mineralogía. Madrid: Gustavo Giti.

KOSTOV, A. (1968). Mineralogy. Londres: Oliver and Boyd.

LILLO, J. (1994), Los trabajos prácticos de ciencias naturales como actividad reflexiva, crítica y creativa. Alambique 2 , pp. 47-56.

MONNET, A.G. (1779). Nouveau système de Minéralogie... París.

NIELSEN, H. y THOMSEN, P. (1990). History and Philosophy of Science in Phisics Education. Int. J. Sc. Education, 12(3), pp. $308-316$.

ORTEGA, P. et al. (1993). La formación de actitudes positivas hacia el estudio de las ciencias experimentales. Revista de Educación, 301, pp. 167-196.
OSBORNE, R. y WITTROCK, M.C. (1983). Learning Science: a generative process. Science Education, 67(4), pp. $489-508$.

PALISSY, B. (1580). Des Pierres (citado en Monnet, 1779).

PIAGET, J. (1970). Piaget's theory, en Mussen, P.H. (ed.). Carmichael's manual of child Psychology. Nueva York: Wiley.

PLINIO (c 75): Naturae historiarum xxxvH libri, (Ed. Desanges, 1., 1950: Histoire naturelle. París: Belles Lettres).

POSNER, G. et al. (1982). Accomodation of a scientific conception: Toward a theory of conceptual change. Science Education, $66(2)$, pp. $211-217$.

POZO, J.I (1989). Teorías congnitivas del aprendizaje. Madrid: Morata.

POZO, J.I. (1993). Psicología y didáctica de las ciencias de la naturaleza, concepciones alternativas? Infancia $y$ Aprendizaje, 62-63, pp. 187-204.

POZO, J.I. et al. (1991): Procesos congnitivos en la comprensión de la ciencia: las ideas de los adolescentes sobre la química. Madrid: MEC.

SALTIEL, E. y VIENNOT, L. (1985). ¿Quế aprendemos de las semejanzas entre las ideas históricas y el razonamiento espontáneo de los estudiantes? Enseñanza de las Ciencias. 3(2), pp. 137-144.

SHAYER, M. y ADEY, P. (1986). La ciencia de enseñar ciencias (desarrollo cognitivo y exigencias del currículo). Madrid: Narcea.

STENON, N. (1669). Prodromus: De solido intra solidum... Florencia.

VYGOSTKI, L.S. (1979). El desarrollo de los procesos psicológicos superiores. Barcelona: Crítica.

WERNER, A.G. (1791). Nueva teoría de la formación de los filones. (Citado en Adams, 1954.)

WHITEN, D.G.A. y BROOKS, J.R.V. (1978). Dictionary of Geology. Harmondsworth: Penguin Books.

WHITEN, D.E.A. y BROOKS, J.R.V. (1980). Diccionario de Geología. Madrid: Alianza.

[Artículo recibido en diciembre de 1995 y aceptado en junio de 1997.] 